\title{
Umklapp-assisted electron transport oscillations in metal superlattices
}

\author{
S. I. Kulinich, ${ }^{1,2}$ L. Y. Gorelik, ${ }^{3, *}$ S. A. Gredeskul, ${ }^{4}$ R. I. Shekhter, ${ }^{2}$ and M. Jonson ${ }^{2,5,6}$ \\ ${ }^{1}$ B. Verkin Institute for Low Temperature Physics and Engineering of the National Academy of Sciences of Ukraine, 47 Lenin Avenue, \\ Kharkov 61103, Ukraine \\ ${ }^{2}$ Department of Physics, University of Gothenburg, SE-412 96 Göteborg, Sweden \\ ${ }^{3}$ Department of Applied Physics, Chalmers University of Technology, SE-412 96 Göteborg, Sweden \\ ${ }^{4}$ Department of Physics, Ben Gurion University of the Negev, P.O. Box 653 Beer-Sheva, 84105, Israel \\ ${ }^{5}$ SUPA, Department of Physics, Heriot-Watt University, Edinburgh EH14 4AS, Scotland, United Kingdom \\ ${ }^{6}$ Department of Physics, Division of Quantum Phases and Devices, Konkuk University, Seoul 143-701, Korea
}

(Received 23 November 2011; published 3 May 2012)

\begin{abstract}
We consider a superlattice of parallel metal tunnel junctions with a spatially nonhomogeneous probability for electrons to tunnel. In such structures tunneling can be accompanied by electron scattering that conserves energy but not momentum. In the special case of a tunneling probability that varies periodically with period $a$ in the longitudinal direction, i.e., perpendicular to the junctions, electron tunneling is accompanied by umklapp scattering, where the longitudinal momentum changes by a multiple of $h / a$. We predict that as a result a sequence of metal-insulator transitions can be induced by an external electric or magnetic field as the field strength is increased.
\end{abstract}

DOI: 10.1103/PhysRevB.85.184202 PACS number(s): 72.15.Rn, 71.30.+h, 73.63.Nm, 85.35.Gv

\section{INTRODUCTION}

To design novel electrical conductors in the form of artificially structured materials remains one of the most important tasks of nanoscience. This is because progress in this type of "quantum engineering" may lead to new and better electronic devices. Multilayered systems are a widely used material of this type, with semiconductor superlattices arguably the most prominent example. ${ }^{1}$ Work on semiconductor superlattices with spatially modulated properties on the submicrometer scale started already 40 years ago, following the pioneering work of Esaki and Tsu. ${ }^{2}$ The early focus on semiconductors was natural, since the de Broglie wavelength of their conduction electrons is typically large enough to be comparable to the period of then feasible superlattices. Qualitatively new effects based on quantum interference phenomena-still mostly absent in metal superlattices ${ }^{3}$ - could therefore be predicted and observed. ${ }^{1}$

More recent developments have led to engineered conductors such as quantum dots, nanowires, and other "nanosolids," which could be useful components in novel superlattice architectures. ${ }^{4}$ Here, we focus on nanowires and note that many are good metals with a high conductivity due to ballistic electron transport. However, the de Broglie wavelength of their electrons is typically much too small compared to the modulation period $a$ of a nanowire-based superstructure for quantum interference effects to occur. Nevertheless, we show below that a prominent interference effect of a different origin emerges in such structures under the realistic assumption that $k_{F} a \gg 1$, so that the quasiclassical approximation is valid and hence the electron energy dispersion can be linearized.

The two-dimensional (2D) superlattice structure to be considered is sketched in Fig. 1. It comprises a set of 1D wires coupled by electron tunneling in such a way that the probability for tunneling varies periodically along the direction of the wires. For this structure we will show that when a magnetic field is applied perpendicular to the $2 \mathrm{D}$ plane-or when an electric field is applied in the plane and perpendicular to the wires - a series of metal-insulator transitions occurs with respect to the interwire hopping transport of electrons as the strength of the external field increases.

Let us start with a single, isolated wire, where electrons move freely along the longitudinal direction and occupy a 1D band of "longitudinal" energies. In the transverse direction, they are confined to the wire and occupy a single, discrete "transverse" energy level. Now, disregard for a moment the longitudinal motion and focus on the transverse electron dynamics in a set of identical wires, aligned in parallel and each with the same transverse level occupied. By switching on a longitudinally uniform probability for electrons to tunnel to adjacent wires, these previously degenerate energy levels will form a band, which allows transverse motion between wires. Hence, in the absence of an external field the superlattice is effectively a two-dimensional (nonisotropic) metal.

If we now apply an external electric field $\overrightarrow{\mathcal{E}}$ perpendicular to the wires, the transverse energy levels will be shifted out of resonance so that band motion in the transverse direction is prevented by Wannier-Stark localization of the electron states. ${ }^{5}$ However, if the tunneling probability can be made to vary periodically along the wires, the situation is qualitatively modified. This is because (i) the longitudinal and transverse motions of the electrons can no longer be separated, and (ii) the longitudinal momentum has to be conserved only modulo $\hbar G$, where $G=2 \pi / a$ and $a$ is the modulation period. Although the total energy is still conserved when electrons tunnel between wires, energy can now be shifted from the longitudinal to the transverse motion in umklapp processes that involve discrete changes of longitudinal momentum. It follows that the transverse energy-level shifts can be compensated and band motion restored for a discrete set of electric field values.

The effect of umklapp-assisted resonant tunneling is controlled by the dimensionless parameter $\phi=\Phi / \Phi_{0}$, where $\Phi=H_{\text {eff }} a b$ is the flux of an "effective" magnetic field $H_{\text {eff }}=$ $\left(c / v_{F}\right)|\overrightarrow{\mathcal{E}}|$ through a superlattice "unit cell" of area $a b$ (see Fig. 1) and $\Phi_{0}=h c / e$ is the magnetic flux quantum. The result 


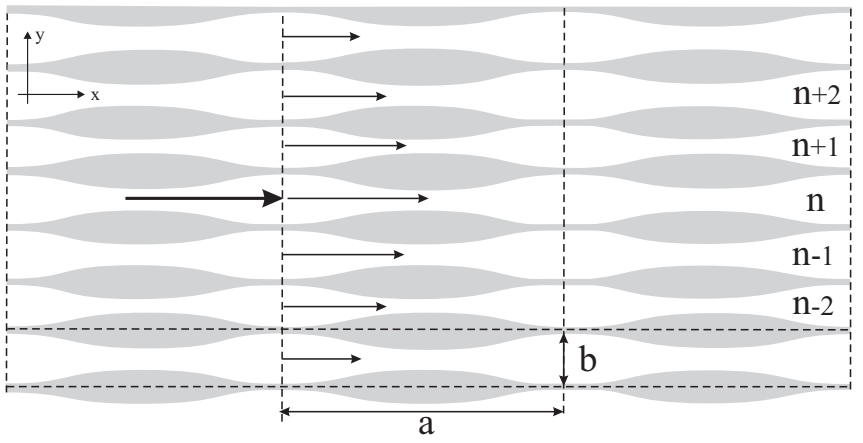

FIG. 1. Sketch of the considered 2D superlattice, comprising an array (period $b$ ) of parallel nanowires coupled by interwire electron tunneling with a periodically modulated tunneling strength $($ period $a)$.

is the same if instead a magnetic field $H$ is applied, except that now $\Phi=H a b$. Resonant tunneling occurs when $\phi=p / q$ is a rational number ( $p$ and $q$ are integers) but, as we will discuss below, band motion is possible only when $\phi$ is an integer (i.e., for $q=1$ ).

In Fig. 2 we illustrate the effect of umklapp-assisted resonant tunneling when $\phi=1$ [Fig. 2(a)] and $\phi=1 / 2$ [Fig. 2(b)]. While in case (a) the resonance condition is fulfilled for neighboring wires, in case (b) every second wire is in resonance and resonant tunneling occurs via virtual states on

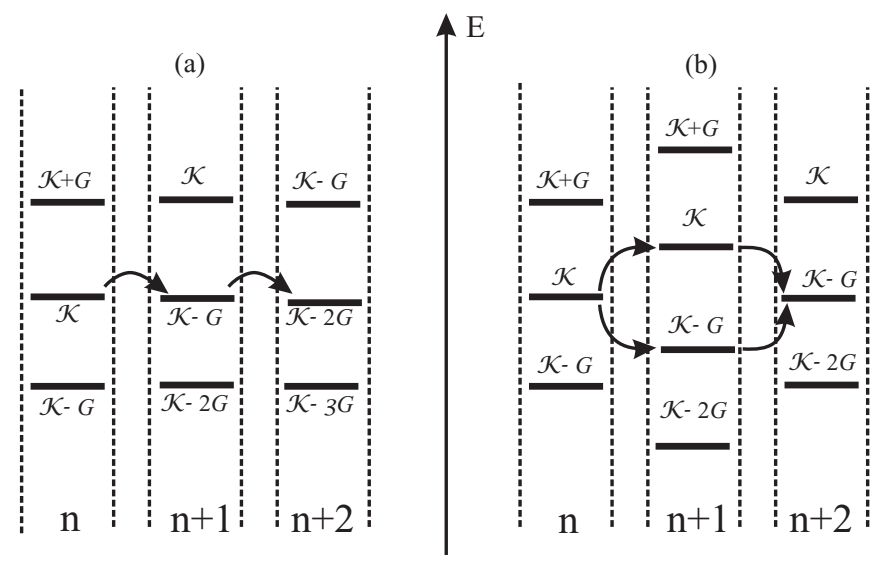

FIG. 2. Transverse energy levels involved in resonant interwire tunneling (thick bars) in three neighboring wires $(n, n+1$, and $n+2)$. The total electron energy is the sum of a longitudinal part proportional to $\mathcal{K}$ (linear spectrum assumed) and a transverse part, which (for fixed $\mathcal{K}$ ) is shifted from one wire to the next by an amount proportional to the dimensionless flux $\phi$ of an external electric or magnetic field. Since $\mathcal{K}$ in the periodically modulated superlattice system of Fig. 1 is conserved only modulo $G$, energy can be shifted between the longitudinal and transverse parts in a tunneling event that conserves the total energy. This is why a sequence of transverse levels is shown for each wire. For integer values of the flux parameter, as in (a) where $\phi=1$, resonant transmissions (arrows) between states on adjacent wires are energetically allowed. In (b), where $\phi=1 / 2$, resonant transitions occur between states on every second wire. The two paths through virtual states on the intermediate wire shown (arrows) contribute with equal amplitudes but opposite signs to to the total transition amplitude, which when all paths are considered turns out to be zero due to destructive interference. the intermediate wire. In this case contributions to the total tunneling amplitude from a number of different paths through various virtual states have to be summed up. The contributions from the two paths identified by arrows in Fig. 2(b) have equal magnitudes but different signs (since the two virtual levels have mirror symmetry with respect to the resonant levels). By generalizing this argument one finds that tunneling between the resonant levels becomes completely suppressed by destructive interference in this case (if direct hopping between next-nearest neighbors is neglected). In the general case of a rational flux, $\phi=p / q$, the transverse energies are resonant for wires separated by a distance $\Delta y=q b$, where $b$ is the superlattice period. Although it would be quite difficult to explicitly consider the destructive interference between all the possible paths for large $q$, we are nevertheless able to prove below that resonant hopping is completely suppressed for any (noninteger) rational value of the parameter $\phi$.

\section{SPECTRAL PROPERTIES}

We consider an infinite superlattice structure (see Fig. 1) subject to a constant electric field $\overrightarrow{\mathcal{E}}=-\mathcal{E} \hat{y}$. In the quasiclassical limit $k_{F} a \gg 1$ large momentum transfers $(\Delta p \sim$ $p_{F}$ ) may be neglected, which justifies a linearization of the energy dispersion related to the longitudinal motion (in each wire). Under this approximation the Hamiltonian is a sum of left- and right-moving electrons, which can be considered independently; the corresponding Hamiltonian of right-moving electrons has the form

$$
\begin{aligned}
\mathcal{H}_{\mathrm{rm}}= & \sum_{n, n^{\prime}} \int d x \Phi_{n}^{\dagger}(x)\left\{-E_{0}\left(i a \frac{\partial}{\partial x}+2 \pi n \phi\right) \delta_{n, n^{\prime}}\right. \\
& \left.+V(x)\left(\delta_{n, n^{\prime}+1}+\delta_{n+1, n^{\prime}}\right)\right\} \Phi_{n^{\prime}}(x) .
\end{aligned}
$$

Here $\Phi_{n}^{\dagger}(x)\left[\Phi_{n}(x)\right]$ is the field operator that creates (destroys) the electron at the point $x$ in the $n$th wire and obeys the standard anticommutation relations; $V(x)=V(x+a)$ is the periodic potential responsible for electron transitions between the wires and $E_{0}=\hbar v_{F} / a$. The same Hamiltonian can be considered in the case of a superlattice subject to a constant magnetic field $\vec{H}=H \hat{z}$. In both cases $\phi$ is the dimensionless flux of the relevant external field - the magnetic field $H$ or the effective magnetic field $H_{\text {eff }}=\left(c / v_{F}\right) \mathcal{E}$-through a unit cell of the superlattice structure.

It follows that the spectrum $E$ (measured from the Fermi level) and the wave functions of the system can be found from the equation

$$
\begin{aligned}
& -E_{0}\left(i \frac{\partial}{\partial x}+2 \pi \phi n\right) \varphi_{n}(x)+v(x)\left[\varphi_{n+1}(x)+\varphi_{n-1}(x)\right] \\
& =E \varphi_{n}(x)
\end{aligned}
$$

where we have introduced a dimensionless coordinate by letting $x / a \rightarrow x, V(a x) \rightarrow v(x)$. Hence, $v(x)$ is the periodic function with unit period.

We will now proceed by solving Eq. (2) exactly for an arbitrary tunneling amplitude $v(x)$. The first step is to note that according to Bloch's theorem $\varphi_{n}(x)=\exp (i \mathcal{K} x) u_{n}(x)$, 
where $\mathcal{K}$ is the (dimensionless) quasimomentum, $-\pi<\mathcal{K}<$ $\pi$, and $u_{n}(x)=u_{n}(x+1)$ is a periodic function. Hence, the auxiliary function $u(n) \equiv u_{n}(x=-1 / 2)$ obeys the equation (see Appendix A),

$$
u(p)=e^{i(\varepsilon-\mathcal{K}+2 \pi \phi p)} \sum_{n} e^{-i \theta(p-n)} J_{p-n}(A) u(n),
$$

where $J_{n}(x)$ is a Bessel function, $\varepsilon=E / E_{0}$, while $A$ and $\theta$ are defined through the relations

$$
\frac{2}{E_{0}} \int_{-1 / 2}^{1 / 2} d y v(y) e^{2 \pi i \phi y} \equiv A e^{i \xi}, \theta=\pi / 2+\pi \phi+\xi .
$$

Equation (3) determines the energy spectrum and the wave functions in our problem. The structure of the spectrum strongly depends on the nature of the number $\phi$. If $\phi$ has a noninteger value, the eigenenergies and eigenstates are labeled by the three quantum numbers $\mathcal{K}, m$, and $r$; the quasimomentum $\mathcal{K}$ and the (integer) band index $m$ refer to the longitudinal motion along the $x$ axis while the integer $r$ is related to the transverse motion in the $y$ direction. The dispersion law reads

$$
E_{m, r}(\mathcal{K})=E_{0}(\mathcal{K}+2 \pi m-2 \pi \phi r), m, r=0, \pm 1, \ldots
$$

The energy-level distribution depends crucially on whether or not the noninteger $\phi$ is a rational number. If it is a rational number, $\phi=p / q$, one notes that $E_{m, r}(\mathcal{K})=E_{m+M p, r+M q}(\mathcal{K})$ for a given quasimomentum $\mathcal{K}$ and any integer $M$. This results (for each $\mathcal{K}$ ) in a set of infinitely degenerate, equidistant energy levels. If $\phi$ is an irrational number, on the other hand, the energy levels are homogeneously distributed, forming a discrete spectrum that is said to be everywhere dense.

The eigenfunctions can be found from Eq. (3) rewritten as

$$
u_{m, r}(n)=e^{-i \xi(n-r)} J_{n-r}(\gamma), \quad \gamma=\frac{A}{2 \sin \pi \phi},
$$

and have the same form for both rational and irrational values of $\phi$. Therefore, for any noninteger $\phi$, all states are localized near a particular wire $r$, within a localization radius $R_{\text {loc }}$ defined as

$$
\left(\frac{R_{\mathrm{loc}}}{b}\right)^{2} \equiv \sum_{n} n^{2} J_{n-r}^{2}(\gamma)-\left[\sum_{n} n J_{n-r}^{2}(\gamma)\right]^{2}=\frac{\gamma^{2}}{2} \text {. }
$$

It is remarkable that this result holds even when the flux parameter $\phi$ is a (noninteger) rational number, since in this case our superlattice structure has translational symmetry in the $y$ direction. Accordingly, bands of electron states with infinite extension in this direction should form (see, e.g., Ref. 6). However, even though the transverse energy levels of the parallel wires periodically are in resonance, this does not happen. The reason is a fully destructive interference between the probability amplitudes for resonant hopping along different paths, as illustrated for the special case of $\phi=1 / 2$ in Fig. 2(b). ${ }^{7}$

In case $\phi$ is an integer, not only does the external field become effectively periodic in the $y$ direction but a band of extended states also forms $\left[R_{\mathrm{loc}} \rightarrow \infty\right.$, according to Eqs. (6) and (7)]. Therefore, in addition to the two longitudinal quantum numbers $\mathcal{K}, m$ a continuous quasimomentum $\tilde{\mathcal{K}}$, where $-\pi<\tilde{\mathcal{K}}<\pi$, must be used to label the transverse motion along the $y$ axis. The dispersion law, which is found from Eq. (3), reads

$$
E_{m}(\mathcal{K}, \tilde{\mathcal{K}})=E_{0}(\mathcal{K}+2 \pi m+A \sin (\tilde{\mathcal{K}}+\theta)) .
$$

Thus, for each pair $(\mathcal{K}, m)$ of eigenvalues related to the longitudinal motion, the transverse energies spread into a band of width $\delta E=2 A E_{0}$. The corresponding eigenstates are of the plane-wave type, $u_{\tilde{\mathcal{K}}, m}(n)=\exp (-i \tilde{\mathcal{K}} n)$, and, as a consequence, are delocalized in both the longitudinal and transverse directions.

\section{ELECTRICAL CONDUCTIVITY}

The detailed features of the energy spectrum influence various physical quantities in essential ways. Here, we consider the linear response of the system to a weak ac external electrical field $\overrightarrow{\mathcal{E}}(t)=\mathcal{E}_{0} \cos \omega t \hat{y}$. The total Hamiltonian of the system $\mathcal{H}=\mathcal{H}_{\mathrm{rm}}+\mathcal{H}_{\text {int }}(t)$ is the sum of the unperturbed Hamiltonian $\mathcal{H}_{\mathrm{rm}}$, Eq. (1), and the perturbation Hamiltonian $\mathcal{H}_{\text {int }}(t)=\mathcal{H}^{(\text {int })} \cos \omega t$, where

$$
\mathcal{H}^{(\text {int })}=e b \mathcal{E}_{0} \sum_{n} n \int d x \Phi_{n}^{\dagger}(x) \Phi_{n}(x) .
$$

Absorption of the ac electric field is proportional to the real part of the conductivity $\sigma(\omega)$, which in linear response theory has the form

$$
\sigma(\omega)=\frac{1}{\mathcal{E}_{0} L} \sum_{\alpha, \beta} I_{\alpha, \beta} \mathcal{H}_{\beta, \alpha}^{(\mathrm{int})} \frac{f\left(E_{\alpha}\right)-f\left(E_{\beta}\right)}{E_{\alpha}-E_{\beta}-\hbar(\omega-i \nu)} .
$$

Here $v$ is a phenomenological relaxation rate, $L$ is the sample length in the longitudinal direction, $f(E)$ is the Fermi-Dirac distribution function, and $I_{\alpha, \beta}$ is the matrix element of the current operator

$$
\hat{I}=\frac{i e}{\hbar N} \sum_{n} \int d x v(x)\left[\Phi_{n+1}^{\dagger}(x) \Phi_{n}(x)-\text { H.c. }\right],
$$

where $N$ is the total number of wires in the superlattice.

Standard calculations lead to an electrical conductivity of the form (see Appendix B)

$$
\begin{aligned}
\sigma(\omega)= & \frac{i e^{2}}{h} \frac{a b}{\left(\hbar v_{F}\right)^{2}} \sum_{n}\left|v_{n}\right|^{2}\left[\frac{1}{2 \pi(n-\phi)-t_{0}(\omega-i \nu)}\right. \\
& \left.-\frac{1}{2 \pi(n-\phi)+t_{0}(\omega-i \nu)}\right]
\end{aligned}
$$

where $t_{0}=a / v_{F}$ and $v_{n}$ is a Fourier component of the potential $v(x)$. In the low-frequency limit, $\omega \ll v$, the electrical conductivity can be approximated as

$$
\sigma(0)=G_{0} \frac{a b}{\left(\hbar v_{F}\right)^{2}} \sum_{n}\left|v_{n}\right|^{2} \frac{t_{0} v}{[2 \pi(n-\phi)]^{2}+\left(t_{0} v\right)^{2}},
$$

where $G_{0}=2 e^{2} / h$ is the conductance quantum.

It follows that in this limit the conductivity plotted as a function of the number of flux quanta per unit cell in the superlattice, $\phi$, has a set of peaks corresponding to integer numbers, $\phi=0, \pm 1, \pm 2, \ldots$ (see Fig. 3 ). The scale of the 


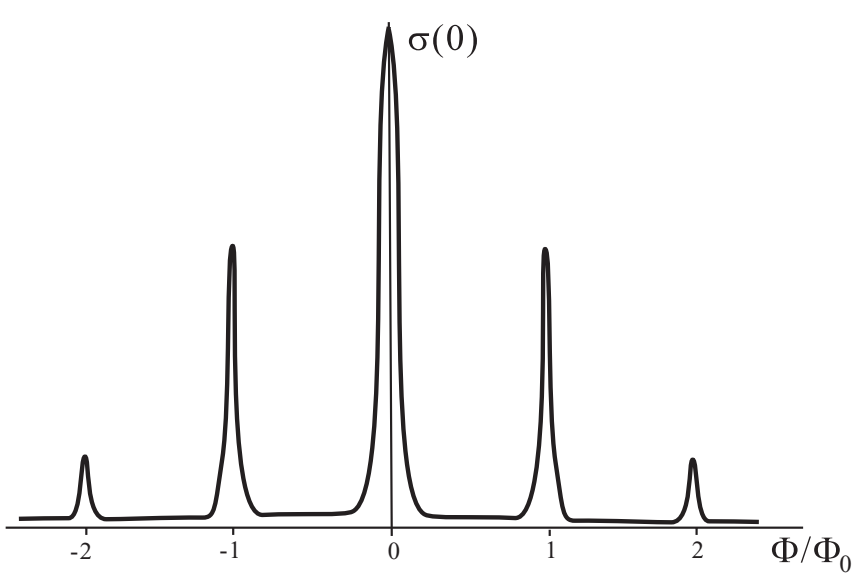

FIG. 3. Schematic behavior of the low-frequency transverse conductivity $\sigma(0)$ as a function of magnetic field flux $\Phi$ through a unit cell of the superlattice structure shown in Fig. $1 ; \Phi_{0}=h c / e$ is the flux quantum.

fluctuations is determined by the ratio between successive $\operatorname{maxima}\left[\sigma_{l}(0), \phi=l\right]$ and minima $\left[\sigma_{l+1 / 2}(0), \phi=l+1 / 2\right]$ of the conductivity,

$$
\frac{\sigma_{l}(0)}{\sigma_{l+1 / 2}(0)} \sim \frac{1}{\left(t_{0} \nu\right)^{2}}, \quad \sigma_{l+1 / 2}(0) \sim G_{0} t_{0} v\left(R_{\mathrm{loc}} / b\right)^{2}
$$

where $R_{\text {loc }}$ is the localization radius, Eq. (7). Hence, if $t_{0} v \ll 1$, the field dependence of the absorption as well as the conductivity has a pronounced peak structure as schematically shown for the conductivity in Fig. 3.

Our analysis ignores any kind of disorder. It is known that in low-dimensional systems disorder leads to a localization of electronic states even in zero external field. In our system localization will be induced by both disorder and the homogeneous external field. The importance of each effect can be characterized by a localization radius and kinetic phenomena will obviously be mostly governed by whichever radius is smaller. It is important that the predicted structure in the microwave absorption will survive even if disorder dominates since the localization radius induced by the field varies periodically with the field strength. However, the intensity of the peaks will be determined by the disorder-induced localization radius rather then by the strength $\left|v_{n}\right|^{2}$ of the umklapp-assisted tunneling processes [Eq. (13)].

Now let us discuss the approximations used in our analysis and estimate the range of system parameter values and external field strengths for which it is valid. First, we have used the Schrödinger equation (2), which has a linear energy spectrum. For this approximation to be valid the longitudinal momentum fluctuations associated with umklapp-assisted resonant transmission of electrons between adjacent wires must be small on the scale of the Fermi momentum $p_{F}$. The total momentum change due to such processes is restricted by the relaxation time $\tau_{r} \sim 1 / v$, which corresponds to coherent tunneling through $N_{v} \sim v_{t} \tau_{r} / b$ wires, where $v_{t} \sim A b E_{0} / \hbar$ is the electron velocity in the transverse direction. Reasonable estimates for the "tunneling" parameter, $A \sim 10^{-3}$, and for the superlattice periods, $a, b \sim 10 \mu \mathrm{m}$, give an upper limit of $\tau_{r} \sim$ $10 \mathrm{~ns}$ for a relaxation time consistent with an approximately linear spectrum. On the other hand, the criterion that the electron motion is ballistic gives a lower limit for the relaxation time of $\tau_{r} \sim b / v_{t} \sim 0.1 \mathrm{~ns}$.

An additional requirement for the Schrödinger equation (2) to be valid must be fulfilled if an external magnetic field is applied to the system. This is because the cyclotron motion of the electrons can be neglected [and a term quadratic in $H$ dropped from Eq. (2)] only if

$$
H \leqslant \frac{E_{F}}{E_{0}} \frac{\Phi_{0}}{2 \pi a b N_{\nu}} .
$$

For a relaxation time $\tau_{r} \sim 1 \mathrm{~ns}$ this restriction corresponds to $H \leqslant 6 \mathrm{~T}$.

The single-particle approach used in our analysis neglects the Coulomb interaction between electrons. In principle, however, a Coulomb blockade of interwire tunneling might prevent the formation of extended electron states in the transverse direction. ${ }^{8}$ To avoid such a blockade the electrostatic charging energy of the wire, $E_{\mathrm{el}} \sim e^{2} / L$, should be smaller than the width $\delta E \sim A E_{0}$ of the transverse energy band. Using our estimates for the relevant parameters, this corresponds to a lower limit of order $100 \mu \mathrm{m}$ for the length $L$ of the nanowires.

Finally we estimate the required strengths of the external electric and magnetic fields by noting that a dimensionless flux of $\phi=1$ corresponds to $\mathcal{E} \sim 0.05 \mathrm{~V} / \mathrm{cm}$ or $H \sim 0.4 \mathrm{~T}$. Such field strengths can easily be applied in an experiment.

\section{CONCLUSIONS}

In conclusion, we have shown that the transport properties of a superlattice comprising a set of parallel metallic nanowires coupled by tunneling in such a way that the tunneling probability varies periodically along the wires differ greatly from the predictions of linear transport theory. In particular, an electric-field-induced sequence of metal-insulator transitions gives rise to highly nonlinear current-voltage characteristics, while the sensitivity to a magnetic field leads to largemagnitude oscillations of the magnetoconductance. It is important that these phenomena are manifest in comparatively weak external fields. Hence, in relatively weak fields interference phenomena give rise to pronounced mesoscopic features in the transport properties of the studied metallic superlattices. Such superlattice structures could, e.g., be realized using arrays of metallic carbon nanotubes ${ }^{9}$ or nanowires similar to those that have been proposed for memory ${ }^{10}$ and mechanical single-electron transistor ${ }^{11}$ applications.

\section{ACKNOWLEDGMENTS}

Financial support from the Swedish Research Council (VR), the European Commission (Grant No. FP7-ICT-2007-C; Project No. 225955 STELE), and the Korean WCU program funded by MEST/KOSEF (Grant No. R31-2008-000-10057-0) is gratefully acknowledged. S.A.G. and S.I.K. acknowledge the hospitality of the Physics Department, University of Gothenburg. 


\section{APPENDIX A: SPECTRUM AND WAVE FUNCTION FOR INTEGER AND NONINTEGER NUMBERS OF FLUX QUANTA $\phi$}

It is convenient to rewrite Eq. (2) in the equivalent form $\varphi_{p}(x)=e^{i(\varepsilon+\Delta p)\left(x-x_{0}\right)} \sum_{n} \frac{1}{2 \pi} \int_{-\pi}^{\pi} d k e^{-i(p-n) k-i G\left(x, x_{0} ; k\right)} \varphi_{n}\left(x_{0}\right)$

where $\Delta=2 \pi \phi$ and

$$
G\left(x, x_{0} ; k\right)=\frac{2}{E_{0}} \int_{x_{0}}^{x} d y v(y) \cos \left[k-\Delta\left(y-x_{0}\right)\right] .
$$

If we search for a solution of Eq. (A1) of the Bloch type, $\varphi_{n}(x)=\exp (i \mathcal{K} x) u_{n}(x)$, then it follows from Eq. (A1) that the function $u_{n}(x)$ satisfies the equation

$$
u_{p}(x)=e^{i(\varepsilon-\mathcal{K}+\Delta p)} \sum_{n} \frac{1}{2 \pi} \int_{-\pi}^{\pi} d k e^{-i(p-n) k-i G(x, k)} u_{n}(x),
$$

where $G(x, k)=G(x+1, x ; k)$. Equation ( 3$)$ is a consequence of Eq. (A3).

It follows from Eq. (3) that if $u_{0}(n)$ is a solution with $\varepsilon=\varepsilon_{0}$ then $u_{1}(n)=u_{0}(n-1)$ is a solution with $\varepsilon_{1}=\varepsilon_{0}-\Delta$. Therefore, the spectrum has the form

$$
\varepsilon_{r, m}=\varepsilon_{0}+2 \pi m-r \Delta, m, r=0, \pm 1, \pm 2, \ldots,
$$

and the corresponding wave function is $u_{r, m}(n)=u_{r}(n)=$ $u_{0}(n-r)$. As a consequence, one has to determine only one solution $u_{0}(n)$ (together with the corresponding energy value $\left.\varepsilon_{0}\right)$.

To proceed, define the function

$$
W(k)=\sum_{n} u(n) e^{l n(k+\theta)}, W(k+2 \pi)=W(k),
$$

which, as follows from Eq. (3), satisfies the equation

$$
W(k-\Delta)=e^{i\left(\varepsilon_{0}-\mathcal{K}+A \sin k\right)} W(k) .
$$

If we now let $W(k)=\exp [i w(k)], w(k+2 \pi)=w(k)$, then

$$
w(k-\Delta)-w(k)=\varepsilon_{0}-\mathcal{K}+A \sin k .
$$

Integrating the last equation over the period, one gets $\varepsilon_{0}=\mathcal{K}$. The solution of Eq. (A7),

$$
w(k)=\frac{A}{2 \sin \pi \phi} \cos (k+\pi \phi),
$$

determines the corresponding wave function $u_{0}(n)$, Eq. (6), in the case of noninteger $\phi$.

The expression for the spectrum in the case of integer $\phi$, Eq. (8), is a consequence of the well-known identity for Bessel functions,

$$
e^{\imath z \sin \varphi}=\sum_{k} J_{k}(z) e^{\imath k \varphi}
$$

\section{APPENDIX B: CALCULATION OF THE ELECTRICAL CONDUCTIVITY}

To calculate the conductivity we take into account that the commutator between the unperturbed Hamiltonian $\mathcal{H}_{\mathrm{rm}}$ and the perturbation Hamiltonian $\mathcal{H}^{(\text {int })}$ is proportional to the current operator,

$$
\left[\mathcal{H}_{\mathrm{rm}}, \mathcal{H}^{(\mathrm{int})}\right]=\imath \hbar \mathcal{E}_{0} b N \hat{I} .
$$

As a result, the electrical conductivity, Eq. (10), takes the form

$$
\sigma(\omega)=-\imath \hbar b \frac{N}{L} \sum_{\alpha, \beta} \frac{\left|I_{\alpha, \beta}\right|^{2}}{E_{\alpha}-E_{\beta}} \frac{f\left(E_{\alpha}\right)-f\left(E_{\beta}\right)}{E_{\alpha}-E_{\beta}-\hbar(\omega-\imath \nu)} .
$$

Thus the problem is reduced to a calculation of the matrix element of the current operator $\hat{I}$,

$$
\begin{aligned}
I_{\alpha, \beta} & =\frac{l e}{\hbar}\left(h_{\alpha, \beta}-h_{\beta, \alpha}^{*}\right),\{\alpha, \beta\}=(\mathcal{K}, m, r), \\
h_{\alpha, \beta} & =\frac{1}{N} \sum_{n} \int d x v(x)\left[\varphi_{n+1}^{(\alpha)}(x)\right]^{*} \varphi_{n}^{(\beta)}(x) .
\end{aligned}
$$

Using the periodicity of the Bloch functions, the matrix element $h_{\alpha, \beta}$ can be expressed as

$$
\begin{aligned}
& \left\langle\mathcal{K}, m, r|\hat{h}| \mathcal{K}^{\prime}, m^{\prime}, r^{\prime}\right\rangle \\
& \quad=2 \pi \delta\left(\mathcal{K}-\mathcal{K}^{\prime}\right) \frac{1}{N} \sum_{n} \int_{-1 / 2}^{1 / 2} d x v(x)\left[u_{n+1}^{(m, r)}(x)\right]^{*} u_{n}^{\left(m^{\prime}, r^{\prime}\right)}(x) .
\end{aligned}
$$

Notice that the appearance of the $\delta$ function in Eq. (B4) is due to the infinite size of the system; the squared $\delta$ function in the matrix element of the current operator, Eq. (B2), is transformed in the usual manner as

$$
\left[2 \pi \delta\left(\mathcal{K}-\mathcal{K}^{\prime}\right)\right]^{2} \rightarrow \frac{L}{2 \pi a} \delta\left(\mathcal{K}-\mathcal{K}^{\prime}\right) .
$$

The coordinate dependence of the functions $u_{n}^{(m, r)}(x)$ is determined by Eqs. (6), (A1), and (A2) as follows: First, define the functions $\chi(x)$ and $\vartheta(x)$ by the relation

$$
\frac{2}{E_{0}} \int_{-1 / 2}^{x} d y v(y) e^{i \Delta y} \equiv \chi(x) e^{i \vartheta(x)}
$$

Then, with the help of Eqs. (6), (A1), and (A2) the expression for the functions $u_{n}^{(m, r)}(x)$ takes the form

$$
\begin{aligned}
u_{p}^{(m, r)}(x)= & e^{l[2 \pi m+\Delta(p-r)](x+1 / 2)-l(p-r)[\vartheta(x)+\theta-\xi]} \\
& \times \sum_{n} e^{\imath n[\vartheta(x)+\theta-2 \xi]} J_{p-r-n}(\chi(x)) J_{n}(\gamma) .
\end{aligned}
$$

The last expression can be simplified using a "summation theorem" for Bessel functions. ${ }^{12}$ As a result

$$
u_{p}^{(m, r)}(x)=e^{i[2 \pi m+\Delta(p-r)](x+1 / 2)-i(p-r) q_{1}(x)} J_{p-r}\left(q_{2}(x)\right),
$$

where the definite forms of the functions $q_{1,2}(x)$, which do not depend on the quantum numbers $m$ and $r$, are not relevant. Substituting this expression in Eq. (B4), one gets

$$
\begin{aligned}
& \left\langle\mathcal{K}, m, r|\hat{h}| \mathcal{K}^{\prime}, m^{\prime}, r^{\prime}\right\rangle \\
& \quad=2 \pi \delta\left(\mathcal{K}-\mathcal{K}^{\prime}\right) \delta_{r-1, r^{\prime}} \frac{1}{N} \int_{-1 / 2}^{1 / 2} d x v(x) e^{-2 \imath \pi\left(m-m^{\prime}\right)(x+1 / 2)} .
\end{aligned}
$$

As a consequence one gets, with the help of Eqs. (B2) and (B3), for the electrical conductivity the result

$$
\sigma(\omega)=-\frac{l e^{2}}{h} \frac{a b}{\left(\hbar v_{F}\right)^{2}}[R(\phi)+R(-\phi)],
$$


where the quantity $R(\phi)$ is defined as

$$
\begin{aligned}
R(\phi)= & \frac{1}{2 \pi N} \sum_{m, m^{\prime}, r} \frac{\left|v_{m-m^{\prime}}\right|^{2}}{m-m^{\prime}-\phi} \\
& \times \int_{-\pi}^{\pi} d \mathcal{K} \frac{f\left(E_{r, m}(\mathcal{K})\right)-f\left(E_{r-1, m^{\prime}}(\mathcal{K})\right)}{2 \pi\left(m-m^{\prime}-\phi\right)-t_{0}(\omega-\imath \nu)}
\end{aligned}
$$

which can be simplified to read

$$
R(\phi)=-\sum_{n} \frac{\left|v_{n}\right|^{2}}{2 \pi(n-\phi)-t_{0}(\omega-\imath \nu)} .
$$

Substituting this expression into Eq. (B10), one gets the result Eq. (12). *gorelik@chalmers.se

${ }^{1}$ See, e.g., Semiconductor Superlattices, edited by H. T. Grahn (World Scientific, Singapore, 1995).

${ }^{2}$ L. Esaki and R. Tsu, IBM J. Res. Dev. 14, 61 (1970).

${ }^{3}$ The importance of magnetic-metal superlattices is not due to quantum coherence: see, e.g., I. K. Schuller, S. Kim, and C. Leighton, J. Magn. Magn. Mat. 200, 261 (1999).

${ }^{4}$ R. Tsu, Nanoscale Res. Lett. 6, 127 (2011).

${ }^{5}$ See, e.g., Ch. 3 of Ref. 1.

${ }^{6}$ L. Gorelik, S. Kulinich, Yu. Galperin, R. I. Shekhter, and M. Jonson, Phys. Rev. Lett. 78, 2196 (1997); L. Y. Gorelik, S. I. Kulinich, R. I. Shekhter, and M. Jonson, Phys. Rev. B 69, 094516 (2004).
${ }^{7}$ Band motion in the $y$-direction can occur, however, if direct tunneling between non-neigboring wires is allowed.

${ }^{8}$ R. I. Shekhter, Zh. Eksp. Teor. Fiz. 63, 1400 (1972) [Sov. Phys. JETP 36, 747 (1973)]; I. O. Kulik and R. I. Shekhter, Zh. Eksp. Teor. Fiz. 68, 623 (1975) [Sov. Phys. JETP 41, 308 (1975)].

${ }^{9}$ N. A. Melosh, A. Boukal, F. Diana, B. Gerardot, A. Sabolato, P. M. Petroff, and J. R. Heath, Science 300, 112 (2003); J. R. Heath and M. A. Ratner, Phys. Today 56(5), 43 (2003).

${ }^{10}$ T. Rueckes, K. Kim, E. Joselevich, G. Y. Tseng, C.-L. Cheung, and C. M. Lieber, Science 289, 94 (2000).

${ }^{11}$ D. A. Koenig, E. M. Weig, and J. P. Kotthaus, Nat. Nanotechnol. 3, 482 (2008).

${ }^{12}$ I. S. Gradshtein and I. M. Ryzhik, Tables of Integrals, Series and Products (Academic Press, NY, 1965). 